\title{
Recent Advances in Hydrazone-Based Switches
}

\author{
Xinyan Su*, Hui Chen and Qinqin Ma \\ Department of Polymer Materials, Shanghai University, China
}

*Corresponding author: Xinyan Su, Department of Polymer Materials, College of

Materials Science and Engineering, Shanghai University, China.
Received Date: May 23, 2019

Published Date: May 28, 2019

\section{Introduction}

Molecular switches that can undergo reversible switching between two or more different states in response to chemical, electrochemical or photochemical stimuli, have attracted much attention in recent decades due to their promising applications in chemical sensing, photocontrollable biological process and molecular machines [1,2]. Most prevalent switches, including interlocked molecules, azobenzene, spiropyrans, diarylethenes and fulgides, have been widely investigated and employed for the construction of stimuli responsive systems and materials [2-4].

The hydrazone functional group, which is characterized by the $\mathrm{C}=\mathrm{N}-\mathrm{NH}$ - structure, has been attracted a great deal of interest because of its ease of synthesis, modularity and functional diversity $[5,6]$. The configurational isomerization (E/Z isomerization) of the $\mathrm{C}=\mathrm{N}$ double bond, nucleophilicity of the hydrazone nitrogen, electrophilicity of the imine carbon, and acidity of the N-H proton, all these features render hydrazones useful for diverse applications, ranging from biology (specifically pharmacology), materials science to supramolecular chemistry. In recent years, the Aprahamian group has developed a series of hydrazone-based switches that show configurational change upon photo or chemical inputs. Phenyl, naphthyl, or quinolyl groups were designed as stators, and rotors with different $\mathrm{H}$-bond acceptors brought different $\mathrm{E} / \mathrm{Z}$ isomerization behaviors.

In this mini review, we will highlight on the effect of intramolecular $\mathrm{H}$-bond on the $\mathrm{E} / \mathrm{Z}$ isomerization and the novel applications of the hydrazone-based switches.

\section{Effect of Intramolecular H-Bond on E/Z Isomerization of Hydrazine-Based Switches}

Arylhydrazone switches with phenyl, naphthyl, or quinolyl group as stators can be obtained by replacing one of the carbonyl groups in 1,2,3-tricarbonyl-2arylhydrazones with pyridine as a proton acceptor. The intramolecular $\mathrm{H}$-bond showed significant effects on switch properties. The $\mathrm{E} \rightarrow \mathrm{Z}-\mathrm{H}+$ reaction is triggered only when the pyridine is protonated. Compared with phenyl group, the naphthyl group makes the $\mathrm{N}-\mathrm{H}$ proton more acidic and hence the H-bond with the ester carbonyl is stronger, which results in faster reaction rate $[7,8]$. It was found that both electron donating and withdrawing para-substituents strengthen the H-bond in the $\mathrm{HN}-\mathrm{N}=\mathrm{C}-\mathrm{C}=\mathrm{N}$ fragment by inversely modulating the acidity and basicity of the $\mathrm{N}-\mathrm{H}$ and pyridyl nitrogen [9]. Different $\mathrm{R}$ groups in the rotor part also effect on the $\mathrm{E} / \mathrm{Z}$ isomerization of the hydrazonebased switches, only the R group which can offer a second H-bondaccepting site and is a moderate H-bond acceptor enables full isomerization [10]. When imidazolyl ring was incorporated as a rotor, a switching cascade can be achieved through coordinationcoupled proton relays. The electrostatic repulsion between the metal cation and the weakly hydrogen-bonded proton in the nonplanar imidazolium ring lowed the pKa of the imidazolyl ring, which is the crucial step [11]. By introducing an additional intramolecular $\mathrm{H}$-bond in quinolylhydrazone, both configuration and conformation isomerization can be achieved [12]. Exchanging a pyridyl rotor with a phenyl group (not H-bond acceptor) not only lead to efficient photoisomerization but also extraordinary thermal stability of $\mathrm{Z}$ isomer [13].

\section{Applications of Hydrazone-Based Switches}

Functionalized with well-studied chiral mesogen cholesterol, a chemically activated molecular switch can be used in the modulation of the long-range organization and properties of liquid crystals [14]. Attaching an isosorbide scaffold to hydrazone-based photo switches offer a chiral dopant which can be used to drive a liquid crystal phase [15]. Hydrazone-based photo switches can also drive shape transformations in liquid crystal polymer network to give shape persistent actuators [16]. Quinolylhydrazone can be selectively inducing conformational and configurational changes by careful control of the amount of acid. Based on the advantage of this property, a hydrazone-based robotic arm was designed by incorporating the quinoline unit into a rigid platform and using the rotor to control the directional movement of a mechanical 
arm. The bidirectional transporter system can transport cargo 2 $\mathrm{nm}$ [17]. The same scaffold was also used by the same group to build a molecular assembler capable of controlling the absolute stereochemical outcome of addition to an $\alpha, \beta$-unsaturated ester [18]. Quinolylhydrazone can also be switched via coordinationcoupled deprotonation (CCD), which render them possibilities to modulate fluorescence as well as catalyze imine hydrolysis in solution [19] and develop a negative feedback loop [20].

\section{Conclusion}

The hydrazone-based switches can be endowed with promising properties used in different areas by rational design. Getting molecular switches to communicate and work together to build macroscopic smart materials is still a fascinating challenge.

\section{Acknowledgement}

We sincerely thank Natural Science Foundation of Shanghai (16ZR1411300) for financial support.

\section{Conflict of Interest}

No conflict of interest.

\section{References}

1. Zhang JL, Zhong JQ, Lin JD, Hu WP, Wu K, et al. (2015) Towards single molecule switches. Chem Soc Rev 44(10): 2998-3022.

2. Harris JD, Moran MJ, Aprahamian I (2018) New molecular switch architectures. Proc Natl Acad Sci USA 115(38): 9414-9422.

3. Klajn R (2014) Spiropyran-based dynamic materials. Chem Soc Rev 43: 148-184.

4. Natali M, Giordani S (2012) Molecular switches as photocontrollable "smart" receptors. Chem Soc Rev 41(10): 4010-4029.

5. Tatum LA, Su X, Aprahamian I (2014) Simple Hydrazone Building Blocks for Complicated Functional Materials. Accounts of Chemical Research 47(7): 2141-2149.

6. Su X, Aprahmian I (2014) Hydrazone-based switches, metalloassemblies and sensors. Chem Soc Rev 43: 1963-1981.

7. Landge SM, Aprahamian I (2009) A pH Activated Configurational Rotary Switch: Controlling the E/Z Isomerization in Hydrazones. Journal of the American Chemical Society 131(51): 18269-18271.
8. Landge SM, Tkatchouk E, Bentíez D, Lanfranchi DA, Elhabiri M, et al. (2011) A pH Activated Configurational Rotary Switch: Controlling the E/Z Isomerization in Hydrazones. J Am Chem Soc 133(51): 9812-9823.

9. Su X, Lõkov M, Kütt A, Leito I, Aprahamian I (2012) Unusual parasubstituent effects on the intramolecular hydrogen-bond in hydrazonebased switches. Chem Commun 48: 10490-10492.

10. Su X, Lessing T, Aprahamian I (2012) The importance of the rotor in hydrazone-based molecular switches. Beilstein J Org Chem 8: 872-876.

11. Ray D, Foy JT, Hughes RP, Aprahamian I (2012) A switching cascade of hydrazone-based rotary switches through coordination-coupled proton relays. Nature Chemistry 4: 757-762.

12. Su X, Aprahamian X (2011) Switching Around Two Axles: Controlling the Configuration and Conformation of a Hydrazone-Based Switch. Org Lett 31(1): 30-33.

13. Qian H, Pramanik S, Aprahamian I (2017) Photochromic Hydrazone Switches with Extremely Long Thermal Half-Lives. J Am Chem Soc 139(27): 9140-9143.

14. Su X, Voskian S, Hughes RP, Aprahamian I (2013) Manipulating LiquidCrystal Properties Using a pH Activated Hydrazone Switch. Chem Int Ed 52: 1073410739.

15. Moran MJ, Magrini M, Walba DM, Aprahamian I (2018) Driving a Liquid Crystal Phase Transition Using a Photochromic Hydrazone. J Am Chem Soc 140(42): 13623-13627.

16. Ryabchun A, Li Q Lancia F, Aprahamian I, Katsonis N (2019) ShapePersistent Actuators from Hydrazone Photoswitches. J Am Chem Soc 141(3): 1196-1200.

17. Kassem S, Lee AT, Leigh DA, Markevicius A, Solà J (2016) Pick-up, transport and release of a molecular cargo using a small-molecule robotic arm. Nat Chem 8(2): 138-143.

18. Kassem S, Lee ATL, Leigh DA, Marcos V, Palmer LI, et al. (2017) Stereodivergent synthesis with a programmable molecular machine. Nature 549(7672): 374-378.

19. Foy JT, Ray D, Aprahamian I (2015) Regulating signal enhancement with coordination-coupled deprotonation of a hydrazone switch. Chem Sci 6: 209-213.

20. Pramanik A, Aprahamian I (2016) Hydrazone Switch-Based Negative Feedback Loop. J Am Chem Soc 138(46): 15142-15145. 